\title{
S-nitrosylation of E3 ubiquitin-protein ligase RNF213 alters non-canonical Wnt/Ca +2 signaling in the P301S mouse model of tauopathy
}

\author{
Haitham Amal (1) ', Guanyu Gong ${ }^{1}$, Elizabeta Gjoneska², Sarah M. Lewis', John S. Wishnok', Li-Huei Tsai ${ }^{2}$ and
} Steven R. Tannenbaum ${ }^{1,3}$

\begin{abstract}
Mutations in the MAPT gene, which encodes the tau protein, are associated with several neurodegenerative diseases, including frontotemporal dementia (FTD), dementia with epilepsy, and other types of dementia. The missense mutation in the Mapt gene in the P301S mouse model of FTD results in impaired synaptic function and microgliosis at three months of age, which are the earliest manifestations of disease. Here, we examined changes in the Snitrosoproteome in 2-month-old transgenic P301S mice in order to detect molecular events corresponding to early stages of disease progression. S-nitrosylated (SNO) proteins were identified in two brain regions, cortex and hippocampus, in P301S and Wild Type (WT) littermate control mice. We found major changes in the Snitrosoproteome between the groups in both regions. Several pathways converged to show that calcium regulation and non-canonical Wnt signaling are affected using GO and pathway analysis. Significant increase in 3-nitrotyrosine was found in the CA1 and entorhinal cortex regions, which indicates an elevation of oxidative stress and nitric oxide formation. There was evidence of increased Non-Canonical Wnt/Ca++ (NC-WCa) signaling in the cortex of the P301S mice; including increases in phosphorylated CaMKII, and S-nitrosylation of E3 ubiquitin-protein ligase RNF213 (RNF213) leading to increased levels of nuclear factor of activated T-cells 1 (NFAT-1) and FILAMIN-A, which further amplify the NC-WCa and contribute to the pathology. These findings implicate activation of the NC-WCa pathway in tauopathy and provide novel insights into the contribution of S-nitrosylation to NC-WCa activation, and offer new potential drug targets for treatment of tauopathies.
\end{abstract}

\section{Introduction}

Tau protein is associated with several neurodegenerative diseases, including Alzheimer's disease (AD), and different frontotemporal dementias, as well as dementia

\footnotetext{
Correspondence: Haitham Amal (hamal@mit.edu) or

Steven R. Tannenbaum (srt@mit.edu)

'Department of Biological Engineering, Massachusetts Institute of Technology, Cambridge, MA 02139, USA

${ }^{2}$ The Picower Institute for Learning and Memory, Department of Brain and Cognitive Sciences, Massachusetts Institute of Technology, Cambridge, MA 02139, USA

Full list of author information is available at the end of the article.

These authors contributed equally: Haitham Amal, Gunayu Gong
}

following traumatic brain injury ${ }^{1}$. Tau is a member of the microtubule-associated proteins (MAPs) that is located on chromosome $17 \mathrm{q} 21.31$ in humans and coded by the $M A P T$ gene $^{2}$.

Tau's main function is to promote microtubule (MT) assembly and modulate the stability of axonal $\mathrm{MTs}^{3,4}$. Tau is a phosphoprotein and is known to be phosphorylated on Serine and Threonine sites ${ }^{5}$. Tau phosphorylation sites are clustered in regions flanking the MT binding repeats and hyperphosphorylation of tau inhibits MT assembly ${ }^{6,7}$ leading to the formation of distinct aggregates of tau ${ }^{1}$, which constitute neurofibrillary tangles (NFTs) in $\mathrm{AD}^{8}$.

\section{(c) The Author(s) 2019}

(c) (i) Open Access This article is licensed under a Creative Commons Attribution 4.0 International License, which permits use, sharing, adaptation, distribution and reproduction cc) in any medium or format, as long as you give appropriate credit to the original author(s) and the source, provide a link to the Creative Commons license, and indicate if changes were made. The images or other third party material in this article are included in the article's Creative Commons license, unless indicated otherwise in a credit line to the material. If material is not included in the article's Creative Commons license and your intended use is not permitted by statutory regulation or exceeds the permitted use, you will need to obtain permission directly from the copyright holder. To view a copy of this license, visit http://creativecommons.org/licenses/by/4.0/. 
The main dogma in the field is that filamentous tau aggregates are the most destructive and pernicious forms of tau'. Tau also has a major role in axonogenesis, neurite outgrowth $^{10}$, and modulation of the interaction of MTs and actin polymers ${ }^{11}$.

Tau also acts as a scaffold protein that interacts through its amino-terminal projection domain with the Src family tyrosine kinase Fyn ${ }^{12}$, which phosphorylates the NMDAR subunit 2 (NMDAR2). Phosphorylation of NMDAR by Fyn, facilitates the interaction of NMDAR with PSD$95^{13,14}$, leading to NMDAR activation, $\mathrm{Ca}++$ influx, and synaptic excitotoxic downstream signaling ${ }^{15}$. This is important because PSD-95 interacts with neuronal nitric oxide synthase (nNOS) that mediates synaptic association and activation of $\mathrm{nNOS}^{16}$.

S-nitrosylation, the NO-mediated post-translational modification of cysteine thiols (SNO), is known to be involved in different neuropathology, such as $\mathrm{AD}^{17-19}$, Parkinson's ${ }^{20}$ and Huntington's disease ${ }^{18,19}$, and other neurodegenerative disorders ${ }^{21-23}$. Recently and for the first time in the literature, we showed S-nitrosylation involvement in autism spectrum disorder mouse model ${ }^{24}$. SNO regulates the localization and activity of many key enzymes and receptors ${ }^{18,25,26}$ leading to modulation of signaling pathways, synaptic plasticity, axonal elongation, movement of proteins to the cell membrane, and protein assembly $^{18,25}$.

We previously profiled S-nitrosylation in the CK-p25 mouse model of AD, which exhibits DNA damage, aberrant gene expression, increased amyloid- $\beta$ levels, and neuronal and synaptic loss followed by cognitive impairment and tau hyperphosphrylation and aggregation at later stages ${ }^{27}$. Our work on the CK-p25 mouse model showed that there was increased S-nitrosylation of proteins important for synapse function, and metabolism, and correlated with amyloid formation ${ }^{17}$. In the current study we profiled the changes in S-nitrosylation in the P301S tau transgenic ( $\mathrm{Tg}$ ) mouse model which overexpresses the human tau mutation identified in early onset familial FTD ${ }^{28}$. The P301S mice exhibit NFTs in the cortex, hippocampus and amygdala and impairment in spatial learning and memory at six months of age ${ }^{29}$. They also showed impaired synaptic function and microgliosis at three months of age, which are the earliest pathological manifestations in this model ${ }^{29}$. We tested whether there is a convergence of pathways between the CK-p25 mouse that models changes in enzymatic activity in $\mathrm{AD}$, and P301S mouse that models genetic change in $\mathrm{FTD}^{28,30}$.

To detect molecular changes that take place during early stages of disease progression in the P301S model, we tested the mice at two months of age. We used the SNOTRAP approach developed in our laboratories ${ }^{17,31}$ to detect differentially S-nitrosylated proteins in P301S mice and littermate age-matched controls. This was followed by GO and pathway analysis that aggregates observations across multiple proteins to identify commonality in the affected biology. This approach allowed us to identify and characterize key proteins, biological processes, and important signaling pathways that could serve as novel therapeutic targets for treatment of tauopathies.

We identified proteins in the NC-WCa signaling pathway that were differentially S-nitrosylated in the P301S mouse model and, as a consequence, the pathway was over-activated in the cortex of these mice.

The major role of the $\mathrm{NC}-\mathrm{WCa}$ is to regulate calcium release from the endoplasmic reticulum (ER) and stabilize the calcium levels in the cell ${ }^{32,33}$. The non-canonical Wnt signaling pathway is a series of molecular signals initiated by binding of a WNT protein to the frizzled family receptor on the surface of the target cell, followed by propagation of the signal via effectors other than betacatenin $^{32}$. This leads to downstream changes such as phospholipase C (PLC) activation ${ }^{34}$. PLC activation leads to IP3 production which binds to its receptor on the ER resulting in calcium release ${ }^{32}$ and downstream activation of kinases, including calmodulin-dependent protein kinase II (CaMKII). Ca++ release also leads to CaMKII activation and autophosphorylation ${ }^{35}$ and subsequent phosphorylation of NMDAR, which further potentiates $\mathrm{Ca}++$ influx $^{32,36}$ and NC-WCa activation.

The role of Wnt signaling pathways in AD mainly as a neuro-protective mechanism against amyloid beta plaques has previously been investigated, however, the function of the NC-WCa pathway in tauopathy has not yet been examined $^{32,36,37}$. Here we identify key proteins that are potential indicators of early disease manifestation and show for a first time that S-nitrosylation is a mechanism that regulates the function of the NC-WCa pathway, which is disrupted in a tauopathy model of neurodegeneration.

\section{Materials and methods \\ Animals}

All mouse experiments were approved by the Committee on Animal Care of the Division of Comparative Medicine at Massachusetts Institute of Technology (MIT). Two-month-old male tau P301S mice and respective wild type (WT) control mice were used. For tissue dissection, mice were chosen in an alternate order, and similarly, samples preparation was done in an alternate order.

Cortex and hippocampus were collected on ice, immediately flash frozen in liquid nitrogen and stored at $-80^{\circ} \mathrm{C}$.

\section{Materials and reagents}

Biotin-PEG3-propionic acid was purchased from ChemPep Inc. Protease cocktail inhibitors were purchased from Sigma-Aldrich and sequencing-grade modified 
trypsin from Promega. High-performance liquid chromatography (HPLC) and the liquid chromotography-mass spectrometry solvents were HPLC grade. Acetonitrile $(\mathrm{ACN})$ and distilled water for MS use were purchased from Sigma-Aldrich. Vivapsin $10 \mathrm{k}$ molecular weight cutoff (MWCO) filters were purchased from Sartorius Stedim NA. Synthesis of SNOTRAP-biotin and NMR analysis are described in detail by Seneviratne et al. ${ }^{17}$. All sample preparation was conducted in dark conditions at room temperature (RT).

\section{Mass spectrometry (MS) sample preparation}

Tissues were homogenized in a $1 \mathrm{ml}$ (for one intact cortex) or $0.5 \mathrm{ml}$ (for one intact hippocampus) of lysis buffer on ice using a Teflon pestle and a Jumbo Stirrer (Thermo Fisher). The lysis buffer was freshly prepared and contained $250 \mathrm{mM}$ HEPES- $\mathrm{NaOH}(\mathrm{pH} 7.7), 1 \mathrm{mM}$ EDTA, $0.1 \mathrm{mM}$ neocuproine, $8 \mathrm{M}$ urea, $20 \mathrm{mM}$

Iodoacetamide (IAM), and $1 \%$ protease inhibitor cocktail (Sigma-Aldrich, Cat. No. P8340).

The homogenates were centrifuged at $12000 \times g$ for $10 \mathrm{~min}$ at $4{ }^{\circ} \mathrm{C}$ and supernatants were collected. The protein concentration was determined by the Bradford assay (Bio-Rad, Cat. No. 500-0006). Equal amounts of cortical protein $(4 \mathrm{mg})$ from three mice were pooled into one cortex sample; equal amounts of cortex protein $(2 \mathrm{mg})$ from six mice were pooled as the negative sample. After mixing, there were two cortex samples and one negative cortex sample for P301S Tg and WT mice, respectively. In the case of the hippocampus, we pooled $1 \mathrm{mg}$ protein from three mice as one hippocampus sample and $0.5 \mathrm{mg}$ protein from six mice as the hippocampus negative sample.

Negative controls were generated by treatment with $10 \mathrm{mM}$ TCEP for $30 \mathrm{~min}$ at $37^{\circ} \mathrm{C}$ after sample mixing. Samples were then alkylated with $30 \mathrm{mM}$ IAM in the presence of $2.5 \%$ SDS in the dark at $37^{\circ} \mathrm{C}$. After alkylation, samples were washed with three volumes of $8 \mathrm{M}$ urea (in 50 mM HEPES, pH 7.7) twice and 50 mM HEPES (pH 7.7) once followed by centrifugation at $5000 \times \mathrm{g}$ for $30 \mathrm{~min}$ at $4{ }^{\circ} \mathrm{C}$ with $10 \mathrm{~K}$ MWCO spin filters (pre-rinsed with water once, Sartorius Corporation, Cat. No. VS15T01).

SNOTRAP labeling stock solutions (in $40 \%$ acetonitrile) were added to all samples to reach a final concentration of $1.25 \mathrm{mM}$ (in $50 \mathrm{mM}$ HEPES buffer at pH 7.7) to selectively convert SNO to stable disulfide-iminophosphorane. Samples were incubated with SNOTRAP solution at room temperature for $1.5 \mathrm{~h}$. After SNOTRAP labeling, excessive reagents were removed by three washes with $50 \mathrm{mM}$ HEPES, pH 7.7 buffer with $10 \mathrm{~K}$ filters.

After ultrafiltration, $200 \mu \mathrm{l}$ of pre-rinsed Streptavidin agarose beads (Pierce, Cat. No. 20349) were added to each sample and incubated for $1 \mathrm{~h}$ at room temperature with gentle agitation. The beads were washed with washing buffer 1 (50 mM HEPES, $150 \mathrm{mM} \mathrm{NaCl}, 0.1 \%$ SDS, $\mathrm{pH}$ 7.7) three times and then with washing buffer 2 (50 mM HEPES, pH 7.7) three times. Protein was eluted with $10 \mathrm{mM}$ TCEP (in $50 \mathrm{mM}$ HEPES, pH 7.7) and then alkylated with $10 \mathrm{mM}$ IAM. After alkylation, samples were trypsinized (Promega, Cat. No. V5111) at $37^{\circ} \mathrm{C}$ for $4 \mathrm{~h}$ and then desalted with C18 StageTips.

\section{MS analysis}

Peptides were analyzed using the Agilent HPLC-Chip/ MS system, consisting of a micro-autosampler, a capillary and nano flow pump, and the Chip-Cube that interfaces LC modules and the MS instrument. Water $(0.1 \%$ Formic Acid (FA)) and ACN (0.1\% FA) were used as mobile phases $\mathrm{A}$ and $\mathrm{B}$, respectively. Peptide separations were carried out on a Polaris-HR-Chip-3C18 HPLC-Chip (Agilent Technologies, Cat. No. G4240-62030), consisting of a 360-nL enrichment column and a $75 \mu \mathrm{m} \times$ $150 \mathrm{~mm}$ analytical column, both of which were packed with Polaris C18-A, 180 A, 3- $\mu$ m stationary phase. Peptides were loaded onto the enrichment column from the autosampler at a constant flow of $2 \mu \mathrm{l} / \mathrm{min}$ provided by the capillary pump. A 55-min gradient started at $3 \%$ B at $300 \mathrm{nl} / \mathrm{min}$ and increased to $30 \% \mathrm{~B}$ from 2 to $35 \mathrm{~min}$, to $60 \% \mathrm{~B}$ at $40 \mathrm{~min}$, to $90 \% \mathrm{~B}$ at $45 \mathrm{~min}$ and then was held for $5 \mathrm{~min}$ and followed by a $5 \mathrm{~min}$ post-run at 3\% B. MS analysis was performed with an Agilent 6550 Accurate Mass Ion Funnel QTOF Chip-MS System operated in positive-ion mode. MS spectra were acquired in the 1700 Da extended dynamic range mode $(2 \mathrm{GHz})$ using the following settings: ESI capillary voltage, $1960 \mathrm{~V}$; fragmentor, $360 \mathrm{~V}$; Octopole RF peak, $750 \mathrm{~V}$; drying gas, $13 \mathrm{~L} / \mathrm{min}$; drying temperature, $225^{\circ} \mathrm{C}$. Data were acquired at a rate of 6 MS spectra per second and $3 \mathrm{MS} / \mathrm{MS}$ spectra per second in the mass range of $m / z 300-1700$ for MS and 50-1700 for MS/MS and stored in centroid mode. The maximum number of precursors per cycle was 20 , with a threshold of 5000 ions in a precursor abundance-based scan speed in peptide isotope model, with $+2,+3$ and above charge state preference, and with active exclusion after 1 spectrum and released after $0.15 \mathrm{~min}$. Fragmentation energy was applied at a slope of $3.1 \mathrm{~V} / 100 \mathrm{Da}$ with a 1.0 offset for doubly charged precursors, $3.6 \mathrm{~V} / 100 \mathrm{Da}$ with a -4.8 offset for triply and multiply charged precursors. Mass accuracy was maintained by using the internal reference ion $\mathrm{m} / \mathrm{z}$ 1221.9906. Agilent MassHunter Workstation software was used for data acquisition. Two technical runs were conducted for each sample.

\section{MS data processing}

Agilent Spectrum Mill MS proteomics Workbench B.05 was used for peak list generation, database searching, and FDR estimation. Parameters for data extractions were as follows: cysteine carbamidomethylation for fixed 
modification, precursor $\mathrm{MH}+300-8000 \mathrm{Da}$, scan time range $0-200 \mathrm{~min}$, sequence tag length $>1$, merge scans with same precursor $\mathrm{m} / \mathrm{z} \pm 30 \mathrm{~s} \pm 0.05 \mathrm{~m} / \mathrm{z}$, default for precursor charge, and to find $12 \mathrm{C}$ precursor, and MS noise threshold 100 counts. MS/MS spectra were searched against the mouse SwissProt protein database with $\pm 20 \mathrm{ppm}$ precursor ion tolerance and $\pm 50 \mathrm{ppm}$ fragment ion tolerance. The search included variable modifications of methionine oxidation, protein $\mathrm{N}$-terminal acetylation, deamidation of asparagine and fixed modification of cysteine carbamidomethylation. Peptide and protein False Discovery Rate (FDR) was set to $1.2 \%$ and Spectrum Peak Intensity was set to $30 \%$ for peptide and protein identification. The mass spectrometry proteomics data have been deposited to the ProteomeXchange Consortium (http:// proteomecentral.proteomexchange.org) via the PRIDE partner repository with the dataset identifier $<$ PXD010106>."

\section{Bioinformatics and statistics}

The lists of SNO-proteins were submitted to "MetaCore from Thomson Reuters", MetaCore ${ }^{\mathrm{Tm}}$ version 6.34 build 69200. GO processes, Pathway Maps, and Networks were generated. Terms that were below 0.05, following Benjamini corrected FDR, were considered significant. Pathway, $\mathrm{GO}$, and networks figures were generated by Metacore. STRING $^{38}$ was used to analyze the protein-protein interaction of SNO-proteins (http://string-db.org). Highly reliable interactions (score $>0.7$ ) from neighborhood, gene fusion, co-occurrence, co-expression, experiments, databases and text mining lists were kept. The protein interaction network was then illustrated with Cytoscape 3.2.1. Sample sizes were chosen on the basis of preliminary experiments and our experience with similar experiments. Blind-coded experiments were done, in which the researchers who obtained the data were unaware of the specific genotype of mice.

\section{Western blot (WB)}

WB was used to measure levels of proteins. Supernatant of the homogenized tissue was diluted with reducing buffer, Laemmli sample buffer (Biorad, 161-0737) and electrophoresed on Tris-HCL 4-20\% pre-cast linear gradient gel (Biorad, 4561093) and transferred to PVDF membrane (Biorad, 1620174). The membranes were blocked with 5\% milk in TBS-0.05\% Tween 20 for $1 \mathrm{~h}$ at $\mathrm{RT}$, incubated with primary antibody overnight at $4{ }^{\circ} \mathrm{C}$ and, after six washes with TBS-0.5\%Tween 20, the membrane was incubated with a horseradish peroxidaseconjugated secondary antibody for $1 \mathrm{~h}$ at RT. Protein bands were visualized using ECL reagent. The bands were captured using Fluorchem ${ }^{\text {TM }} 8900$ (Alpha Innotech). Primary antibodies were purchased as follow: nNOS (Cell signaling, 4231 S, 1/1000), iNOS (Santa Cruz, C11, 1/500),
CaMKll (Cell Signalling Technology (CST), 3362, 1/1000 dilution), P-CaMKll (Thr286) (CST, 12716, 1/1000 dilution), GAPDH (CST, 21185, 1/1000 dilution), CREB (Invitrogen, MA1-083, 1/500 dilution), Phospho-CREBSer133-(Invitrogen, PA1-4619, 1/1000 dilution), NFAT1 (CST, D43B1, 1/1000 dilution), and FILAMIN-A (CST, 4762, 1/1000 dilution).

Secondary antibodies used were goat-anti-rabbit (Invitrogen, A11036, 1/10000 dilution) and goat-anti-mouse (Invitrogen, A11001, 1/10000 dilution).

Each group contained three biological replicates. Each biological replicate comprised three pooled cortex or hippocampus tissues from three different mice.

\section{Immunohistochemistry (IHC)}

IHC staining of mouse brain sections was performed using the following antibodies: Nitrotyrosine antibody (Millipore, AB5411, 1/200), NeuN antibody (Millipore, MAB377, 1/200), GFAP antibody (Abcam, ab7260, 1/500), Iba1 (Abcam, ab15690, 1/500). Briefly, whole mouse brains were fixed in $10 \%$ formalin, cut into coronal slices and embedded in paraffin blocks. Formalin-fixed paraffinembedded (FFPE) tissue sections were deparaffinized in $100 \%$ Xylene and gradient washed in 100, 90 and $70 \%$ ethanol and $\mathrm{ddH} 2 \mathrm{O}$. Sections were boiled in the antigen retrieval buffer (Dako, $\mathrm{pH}=6.0, \mathrm{~S} 1700$ ) at $95^{\circ} \mathrm{C}$ for $20 \mathrm{~min}$. Sections were blocked with $3 \%$ BSA in PBS-Triton $\mathrm{X} 100(0.3 \% \mathrm{v} / \mathrm{v})$ overnight, incubated with primary antibody, washed, and incubated with Secondary antibody: goat-anti-rabbit (Invitrogen, A11036, 1/500 dilution) and goat-anti-mouse (Invitrogen, A11001, 1/500 dilution) at RT for $1 \mathrm{~h}$. Sections were washed and counterstained with 4,6diamidino-2-phenylindole (DAPI). Each group contained six mice, when each mouse represents one biological sample.

\section{Results}

To detect early molecular changes in the P301S $\mathrm{Tg}$ model, we profiled S-nitrosylation in two brain regions, the cortex and hippocampus, of P301S mice (Cor-Tg and Hip-Tg, respectively) and WT (Cor-WT and Hip-WT) controls, at two months of age (Fig. 1).

\section{The detection of proteins differentially S-nitrosylated in $\mathrm{Tg}$ vs. control mice}

We identified differences in S-nitrosylation between the Tg and WT groups. A higher number of SNO-proteins were detected in Cor-Tg compared to Cor-WT, while there was no major difference in the number of SNOproteins in the hippocampus (See Fig. 1 and Supplementary Figure $1 \mathrm{~A}-\mathrm{D})$. In both regions, the identity of most of the proteins was different between WT and Tg. Protein IDs of the four different groups are presented in Supplementary Table 1. Analysis of the SNO-proteins 


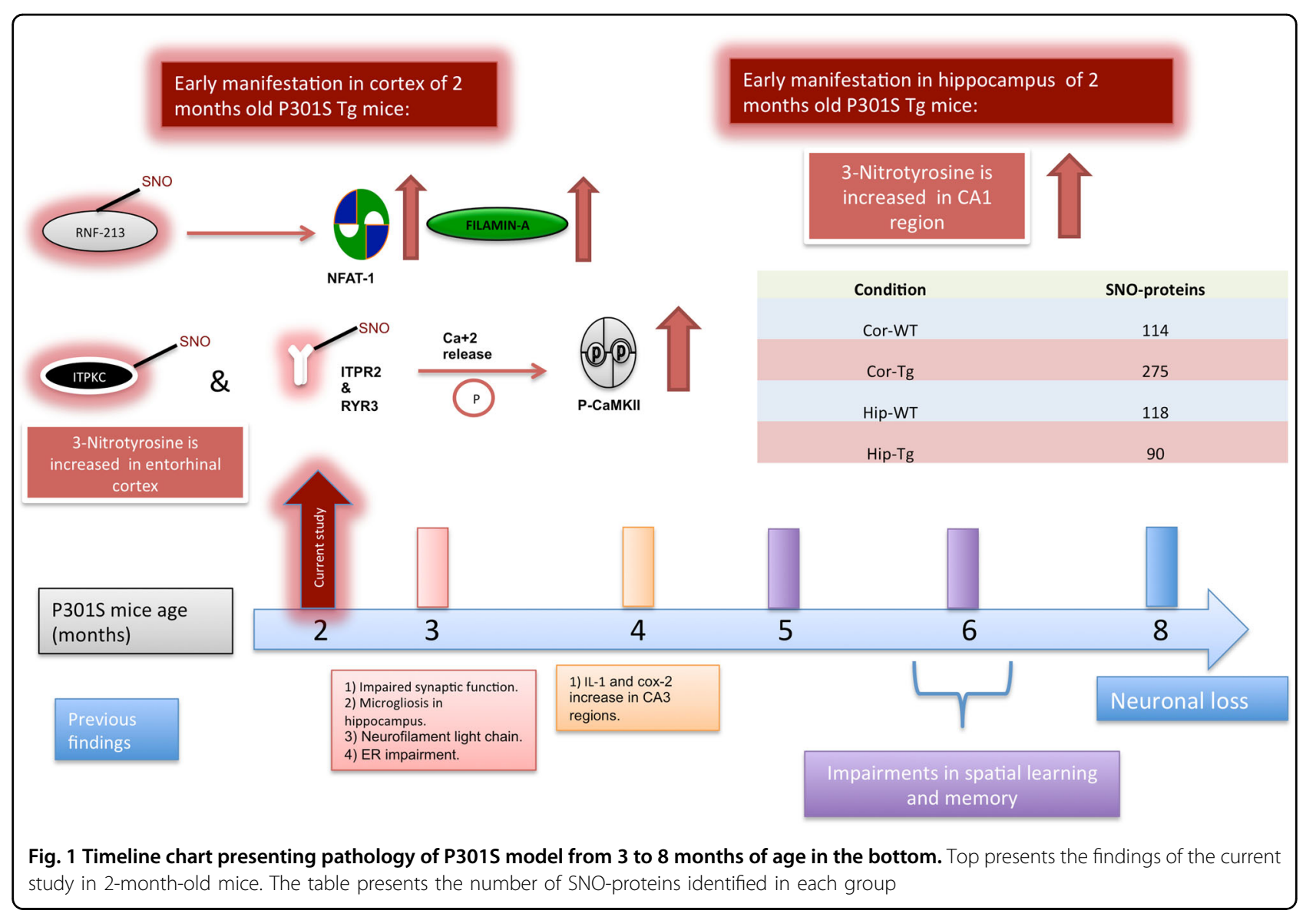

according to function showed a higher number of enzymes, including kinases and transcription factors, that were S-nitrosylated in Cor-Tg compared to Cor-WT (see Supplementary Figure 2).

\section{Gene ontology and pathway analysis of the S- nitrosoproteome}

Our aim in the downstream GO and pathway analysis is to aggregate observations across the SNO-proteins to enable the characterization and identification of (neuro) biological processes and signaling pathways that are affected in tauopathy. GO analysis revealed enrichment of proteins associated with neuronal and synaptic functions, such as nervous system development, neurogenesis, and vesicle-mediated transport, specifically in the cortex of the P301S Tg mouse, but not the WT control (Fig. 2a, Supplementary Table 2). Proteins associated with calcium-related functions such as regulation of calcium ions and calcium homeostasis, were also exclusively enriched in the cortex of the P301S Tg mice. Lastly, components of the non-canonical Wnt signaling pathway were enriched in Cor-Tg mice as well. Specifically we observed S-nitrosylation of the Rac family small Gtpase 1, Frizzled-10 (FZD10), and inositol 1,4,5-trisphosphate receptor type 2 (IP3R2). Consistent with the GO analysis, pathway analysis of Cor-Tg (Fig. 2b) showed enrichment of specific calcium-related pathways, such as signal transduction_IP3 signaling pathway (see Metacore pathway in Supplementary Figure 3) and NMDAR trafficking pathway (see Metacore pathway in Supplementary Figure 4).

GO and pathway analysis of Hip-Tg (Fig. 2c, d respectively and Supplementary Table 3) also showed enrichment of several neuronal and calcium-related processes and pathways, such as response to calcium ions, nervous system development and axon growth (see axon growth pathway by Metacore in Supplementary Figure 5).

These results suggest that neuronal, synaptic, and calcium processes are affected by differential S-nitrosylation in the P301S Tg mice.

\section{Network analysis of S-nitroso-proteome}

Process network analysis was performed to assess overall functional character of the SNO-proteins set in each group. In particular, SNO-proteins in Cor-Tg mice that function in calcium and neuronal processes were clustered in networks enriched for: (1) Non-canonical Wnt signaling pathway $(F D R=4.682 \mathrm{e}-4)$ consisting of FZD10, ITPR2, Rac1, ITPKC, DIAPH2, and others; and (2) Calcium ion homeostasis (5.446E-03) including RYR3, 


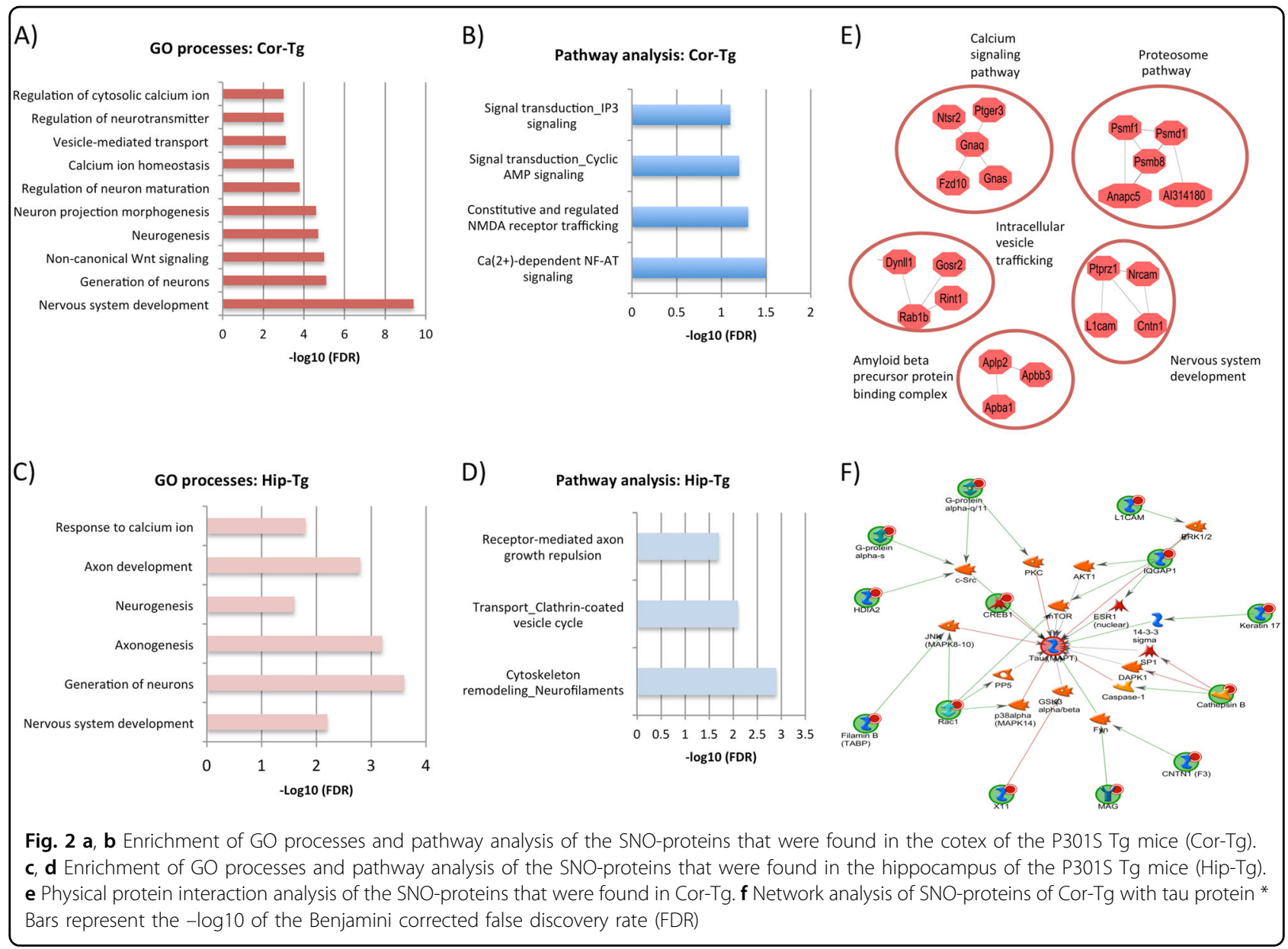

TRPM1, GNAI1, GNAS1 PTGER3, RXFP3, PDE2A, TRPM8, FZD10, TRPM1, IP3R2, GNAQ, P2RX3, ATP1, among others (see Supplementary Table 2). In the hippocampus of the P301S Tg mice, the functional networks showed: (1) Axon development (FDR $=1.596 \mathrm{E}-03)$ that was enriched by WNT11, ADCY3, ULK2, GAP43, TUBB, and others; as well as (2) Response to calcium ion (FDR = 2.249E-04) enriched by WNT11, ADCY3, RYR1, ACTG1, and others (for more information, see Supplementary Table 3).

We used STRING software to analyze protein-protein interactions among S-nitrosylated proteins in the P301S Tg mice. Physical interactomes related to synapse, calcium, and proteasome (Fig. 2e) were generated only in the Cor- $\mathrm{Tg}$ group with "high reliable interactions" score $(>0.7)$, showing a correlation with the functional networks. The network analysis suggests that SNO-proteins are involved in synaptic and calcium processes, as well as ubiquitination (proteosomal) processes. Supplementary Figure 6 shows the interactome analysis of the four tested groups.

Finally, to test the association of the tau protein with the SNO-proteins, we built a network integrating tau using the Metacore software. We identified primary and secondary interactions of tau with the SNO-proteins in the Cor-Tg group (see Fig. 2f).

\section{Quantification of 3-nitrotyrosine (Ntyr) and nNOS in the hippocampal and cortical regions of WT and P301S Tg mice}

As discussed elsewhere ${ }^{39}$, once NO is produced by the intracellular nitric oxide synthase, it undergoes a fast reaction with superoxide anion to form peroxynitrite (OONO-). Peroxynitrite is highly reactive and one of its major targets is tyrosine to which is modified into 3nitrotyrosine $(\mathrm{Ntyr})^{39}$. Therefore, Ntyr level is widely used as indicator of regional NO level. Here, we examined Ntyr levels in various cortical and hippocampal regions using IHC and morphometric quantification of Ntry intensity. This study focused on those brain regions that have functional relevance to the behavioral and physiological deficits in the P301S mouse model: ${ }^{29,40}$ CA1, CA2, CA3, and dentate gyrus regions of the hippocampus as well as the entorhinal cortex (EC). We also tested several other cortical regions known to be relevant in $\mathrm{AD}$, such as the prefrontal cortex, motor cortex and somatosensory cortex $^{41,42}$. 


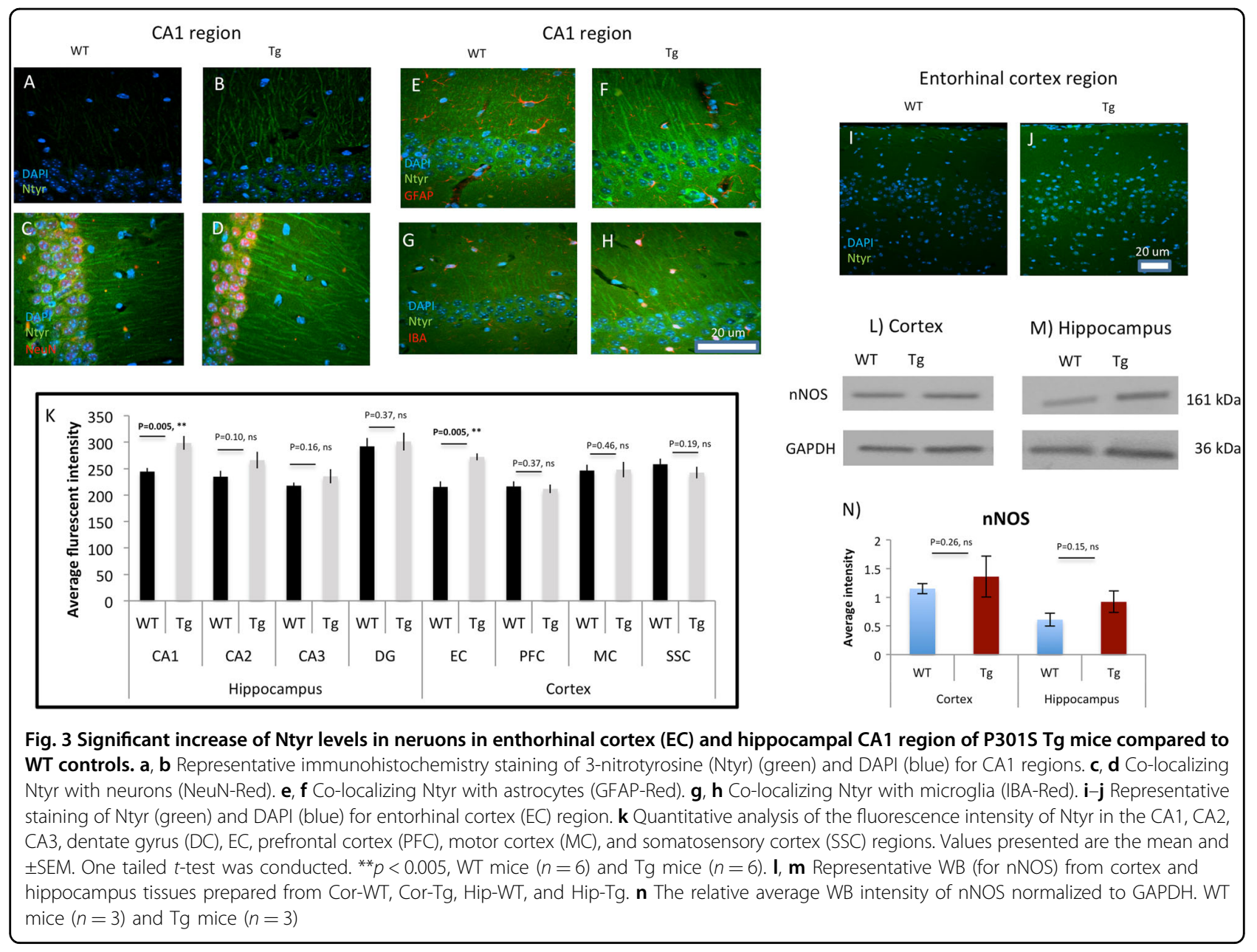

We found a significant increase of Ntyr in the CA1 region ( $p$-value $<0.005)$ in $\mathrm{P} 301 \mathrm{~S} \mathrm{Tg}$ mice compared to WT controls (See Fig. 3a, b, k). A marginal increase was observed in CA2 ( $p$-value $=0.10$; Fig. $3 \mathrm{k}$ ), whereas no difference was detected in the CA3 and DG regions (Fig. 3k). To determine which cell types are the major source of Ntyr in CA1, co-staining experiments were carried out using Ntyr and cell-type specific markers. We found that the vast majority of Ntyr in CA1 is within neurons (Fig. 3c, d) but not in astrocytes (Fig. 3e, f) or microglia (Fig. 3g, h). A similar expression pattern was observed in both WT and P301S Tg mice (Fig. 3c-h).

A significant increase in Ntyr levels was specifically observed in the EC ( $p$-value $<0.005)$ in the P301S Tg mice relative to WT controls (See Fig. 3i-k $)^{43}$.

We examined whether nNOS protein expression changed in the WT compared to Tg groups in both cortex and hippocampus tissues. A marginal increase (not significant) was found in Hip-Tg compared to Hip-WT (See Fig. 3m, n) and in Cor-Tg compared to WT-Tg (See Fig. 3l, n).

To test whether inducible NOS (iNOS) plays a role in the P301S mouse model, we measured its level of expression in the cortex and found no difference between WT and Tg mice (See Supplementary Figure 7).

\section{S-nitrosylation of RNF-213 alters non-canonical $\mathrm{Ca}++/$ Wnt signaling}

Several proteins that regulate the $\mathrm{NC}-\mathrm{WCa}$, including FZD10, RNF-213, inositol-triphosphate 3-kinase C (ITPKC), inositol-triphosphate receptor 2 (ITPR2), rynaodine receptor 3 (RYR3), and guanine nucleotidebinding protein $\mathrm{G}(\mathrm{q})$ subunit alpha (GNAQ) were Snitrosylated in the cortex of the P301S Tg mice.

RNF-213 is an E3 ubiquitin-protein ligase that targets NFAT-1, a downstream transcription factor, and FILAMIN-A, for proteasomal degradation attenuating the NC$\mathrm{WCa}^{44}$. Several studies have previously reported that $\mathrm{S}$ nitrosylation of different ligases, such as RNF-25 and PARKIN, leads to its auto-ubiquitantion and, as a consequence, increases its substrates levels ${ }^{45,46}$. We tested two different RNF-213 substrates, NFAT-1 and FILAMIN-A, and found a significant increase in the levels of both substrates in the cortex of the P301S mice (See Fig. 4a, c-e). No differences in NFAT-1 levels were 


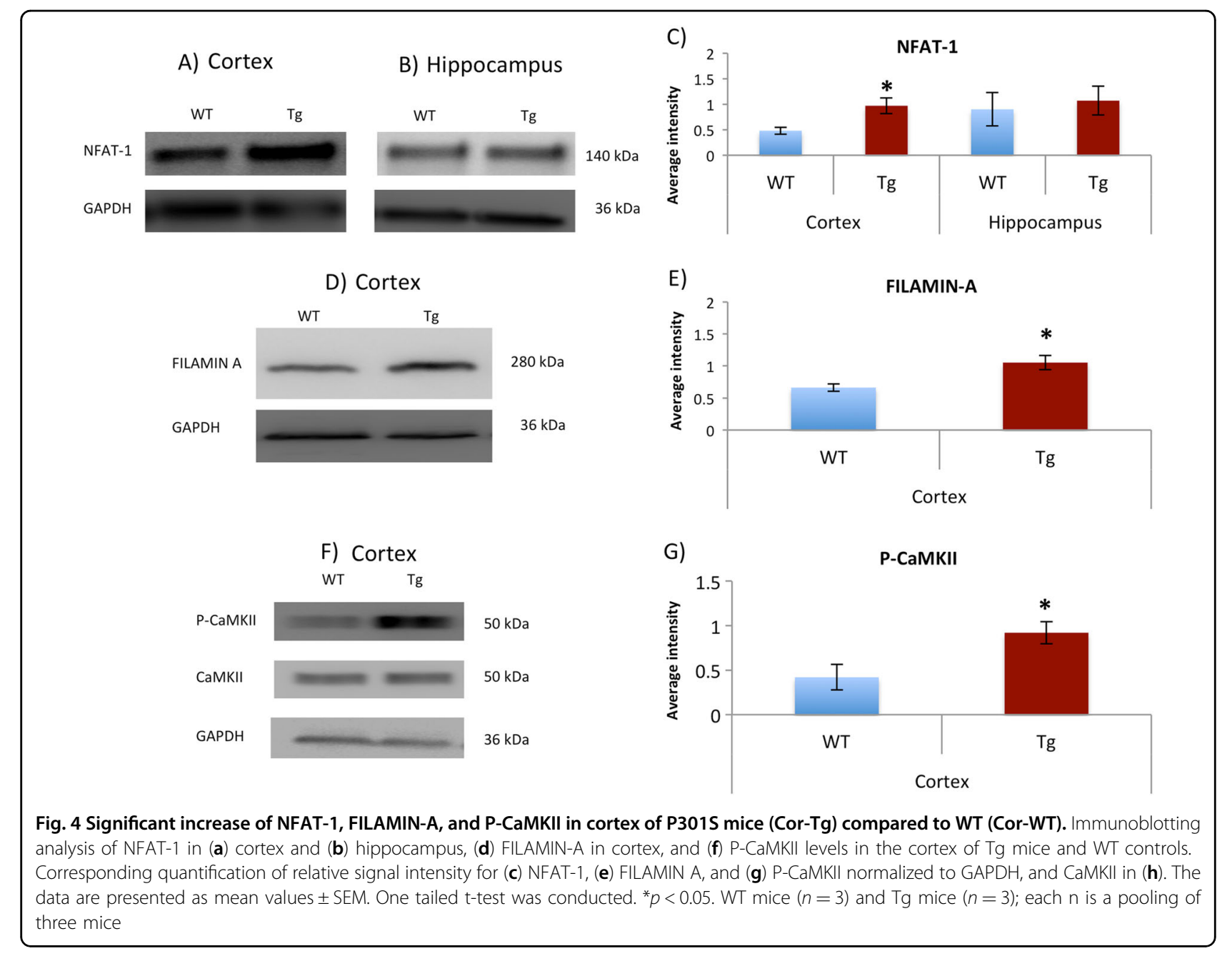

found in the hippocampus region, since SNO-RNF213 was found in the hippocampus of both the P301S Tg mice as well as WT controls (Fig. 4b, c). FILAMIN-A bands were not detected in the hippocampus of both groups.

We examined other components of the pathway for possible alterations. It has previously been reported that calcium leads to activation of the $\mathrm{NC}-\mathrm{WCa}$ via increased auto-phosphorylation of $\mathrm{CaMKII}^{33}$. We found SNOITPKC, SNO-ITPR2, and SNO-RYR3 in Cor-Tg, which may lead to further calcium release and leak from the ER (See Discussion). Therefore we measured phosphorylated CaMKII on threonine 286 as an indication of calcium increase and activation of the NC-WCa pathway. We found a significant increase in P-CaMKII in Cor-Tg (see Fig. 4f, g).

To validate that this enrichment is specific to the NCWCa pathway and not to other non-canonical Wnt pathways, we measured P-CREB, which is not a unique protein for specific Wnt pathway. Supplementary Figure 8 shows that there is no difference between the Tg and WT groups both in P-CREB and CREB, which supports our conclusions.
Furthermore, Supplementary Figure 9 presents the KEGG analysis of three different Wnt signaling pathways and shows the specificity of CaMKll as an upstream protein that activates the NC-WCa pathway. It also shows NFAT as a downstream protein in this pathway and both were increased in our data.

\section{Discussion}

Our study combines state-of-the-art proteomic as well as biological methods to study changes in the S-nitroso-proteome and the consequences on biological processes and signaling pathways in the P301S Tg mouse model of tauopathy. We examined the hippocampus and the cortex of two-month-old mice, which is the earliest age tested in this model (see Fig. 1).

GO and pathway analysis of the S-nitrosoproteome in the P301S Tg mice revealed enrichment of processes and pathways that are known to be affected in $\mathrm{AD}$, pointing to the possibility that S-nitrosylation plays an important role in the neuropathology. For example, in both cortex and hippocampus of the P301S mice, we found enrichment of 
SNO-proteins associated with calcium-related processes (see Fig. 2), indicative of an association between S-nitrosylation, calcium, and neurodegeneration. This correlates with previous findings which support the theory that $\mathrm{Ca}++$ dysregulation plays a major role in $\mathrm{AD}^{47,48}$. Other neuronal, synaptic, and vesicle processes were enriched in either or both regions such regulation of neurotransmitter, neurogenesis, nervous system development, and vesicle mediated transport, which is consistent with results from previous studies showing their involvement in $\mathrm{AD}^{49,50}$.

In the CK-p25-inducible mouse model of $\mathrm{AD}$, proteins related to amyloid precursor protein processing and secretion are S-nitrosylated, correlating with increased amyloid formation and tau aggregates ${ }^{17}$. We compared the results from the CK-p25 to the P310S model to examine whether there is a convergence of processes and pathways (GO and KEGG). Neuronal and calcium related processes were enriched in the cortex of both models, including calcium signaling pathways, neuronal differentiation, neuron projection, and vesicle mediated transport (see Fig. 2). Endosomal pathway abnormalities were previously reported in $\mathrm{AD}^{51,52}$, which is correlated with our findings in both models. This suggests that SNO may be central to $\mathrm{AD}$ pathology, especially in calcium deregulation ${ }^{47,48}$. Since both models showed similarity in behavioral deficits of memory and spatial learning ${ }^{53,54}$, different genetic defects in $\mathrm{AD}$ may lead to common enzymatic deficits that converge into altered biochemical pathways.

Specifically, we found the non-canonical Wnt signaling process was enriched in the GO analysis of the Cor-Tg. Furthermore, other processes related to the non-canonical Wnt pathway were also enriched, such as signal transduction_IP3 signaling (see Supplementary Figure 3) and $\mathrm{Ca}++$ dependent NFAT signaling. The NC-WCa pathway regulates intrinsic properties of neurons, mobilizes intracellular calcium and regulates key components in synapses ${ }^{55}$. Since we found key proteins in the pathway were S-nitrosylated, which may alter the pathway's normal function, we consider their involvement in the NC-WCa in the P301S Tg mice. ITPKC which was S-nitrosylated in the Cor-Tg mice plays an important role in the NC-WCa. ITPKC phosphorylates inositol 2,4,5-triphosphate (IP3) to inositol 2,4,5,6-tetraphosphate (IP4). IP3 acts on IP3R leading to calcium release and activation of the $\mathrm{NC}$ $\mathrm{WCa}^{55}$. Most studies have reported that the effect of Snitrosylation on kinase activity is inhibitory and the inhibition may be exerted directly through suppression of kinase activity or by modulating the interaction of kinase and substrate (reviewed in ${ }^{56}$ ). We therefore suggest that Snitrosylation of ITPKC inhibits its activity and leads to increase in IP3 levels which leads to $\mathrm{Ca}++$ release from the ER (see Fig. 5). Another calcium-related component that was S-nitrosylated in the Cor-Tg is RYR3. A previous investigation has shown that SNO of RYR leads to calcium leak from the $\mathrm{ER}^{57}$, which indicates the possibility that SNO-Ryr3 may also lead to $\mathrm{Ca}++$ leak. $\mathrm{Ca}++$ release in turn has been shown to lead to increase of CaMKII autophosphorylation $^{35}$, which facilitates activation of the $\mathrm{NC}-\mathrm{WCa}$ signaling pathway ${ }^{34}$. Indeed, we observed increased levels of phosphorylated CaMKII consistent with $\mathrm{Ca}++$ dependent activation of the NC-WCa signaling in the P301S Tg mouse. Active CaMKII (P-CaMKII) induces activation of the NFAT-1 which regulates cell adhesion and migration ${ }^{34}$, neuronal growth and axon guidance in both the developing and adult nervous system ${ }^{58}$. Importantly, CaMKII activation also leads to phosphorylation of the tau protein on Thr212, Ser214, Ser262, and Ser356 residue $^{59}$. Another study showed that CaMKII dysregulation in Alzheimer's patients may be a modulator of toxicity in Alzheimer's disease, a dementia characterized by aberrant calcium signaling, synapse and neuronal loss, and impaired memory ${ }^{60}$. A Different study showed that disruption of calcium homeostasis and the downstream kinase CaMKII coincides with pathological phosphorylation of tau in $\mathrm{AD}$ brains ${ }^{61}$.

As previously reported, RNF-213 is inhibited by autoubiquitination upon S-nitrosylation ${ }^{45,46}$, and thus leads to activation of NC-WCa pathway, by reduced turnover of its substrates, NFAT-1 and FILAMIN-A. Indeed, the levels of both NFAT-1 and FILAMIN-A, were increased in the cortex of the P301S mice, consistent with activation of NC-WCa signaling pathway. Our results correlate with previous findings showing accumulation and increased levels of NFAT isoforms in post-mortem brains from patients with $A D$, which further suggest that changes in NFAT levels contribute to AD progression; ${ }^{62}$ The fact that they found increase of NFAT in both early and late stage of the disease suggests that our early molecular findings are linked to the later disease mechanism as well. Other recent studies also showed an increase of NFAT-1 in a different transgenic $A D$ mouse model ${ }^{63}$, and a third proteopathy for $\mathrm{AD}$, suggests that an alteration of the scaffolding protein FILAMIN-A, is critically linked to the amyloid and tau pathologies in $\mathrm{AD}$ and leads to increased tau phosphorylation ${ }^{64}$. Another study showed that FILAMIN-A inhibitors decrease P-Tau and amyloid-beta aggregates $^{65}$. These studies confirm the involvement of NFAT-1 and FILAMIN-A in AD pathology.

Our study demonstrates that S-nitrosylation of key proteins activates the $\mathrm{NC}-\mathrm{WCa}$ signaling pathway as measured by increased phosphorylation of CaMKII in the cortex of the P301S mouse model, and this is likely through increased calcium release from the ER. Moreover, S-nitrosylation of RNF-213 led to an increase in NFAT-1 and FILAMIN-A levels in the cortex of P301S mice, further amplifying the NC-WCa signaling pathway (See Fig. 


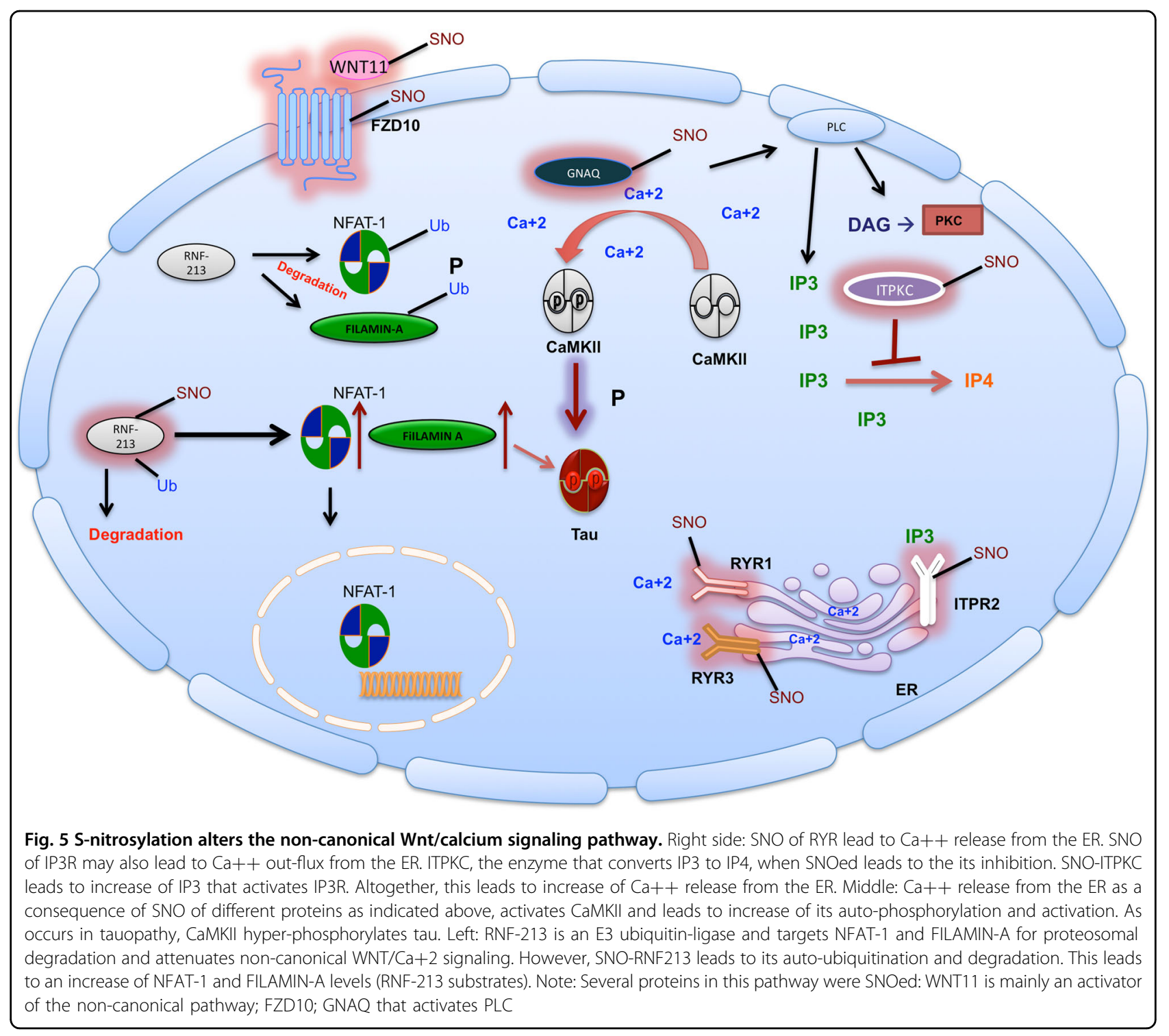

5). Taken together, these findings provide powerful evidence implicating S-nitrosylation mediated alterations of the NC-WCa pathway in tauopathy.

Consistent with the changes in S-nitrosylation we observed a significant increase of Ntyr in CA1 and EC, which indicates an elevation of reactive oxygen species and nitric oxide formation, and has been widely reported in the AD literature (see reviews ${ }^{66,67}$ ). It is also well known that the EC region projects to the CA regions ${ }^{43}$. Moreover CA1 and EC have previously been shown to be a focal point for tau pathology and also the most heavily damaged regions in $\mathrm{AD}^{29}$. Therefore the significant increase of Ntyr in CA1 and EC regions, further validates the specificity of these regions in tauopathay.

We show in Fig. 3 that there may be a marginal, but not statistically significant, increase of nNOS levels in $\mathrm{Tg}$ compared to WT in both cortex and hippocampus.
Further, an increase of free calcium (validated by increase of P-CaMKll) binding to calmodulin could lead to an increase in $\mathrm{NO}$ formation from nNOS. In addition, nNOS is found exclusively in neurons ${ }^{68}$ and proximity is important, because of the high diffusivity and reactivity of NO. To test whether iNOS may play a role in the P301S mouse model, we measured its level of expression in the cortex and found no difference between WT and Tg mice. We favor nNOS as the source of S-nitrosylation in these experiments because it is localized in neurons, but there is no basis for a definitive answer.

In conclusion, we have identified dysregulation of the NC-WCa signaling pathway as an early molecular sign of tau pathology in the cortex of the P301S mouse model. Specifically, we demonstrated that NC-WCa is aberrantly activated in early stages of disease progression. Our study provides a novel insight into the contribution of S- 
nitrosylation to tau pathology and suggests it mediates the dysregulation of NC-WCa. The increase in P-CaMKII, NFAT-1, and FILAMIN-A are correlated with human data discussed above which will allow future translational studies of potential drug targets for treatment of neurodegenerative diseases.

\section{Acknowledgements}

This work was partially funded by the JBP Foundation to L-H T, the MIT Center for Environmental Health Sciences Grant ES002109, the Satell Technion-MIT Post-Doctoral Program for HA, and grant number NIH R01-AG056259 to Stuart Lipton and SRT. We acknowledge Dr. Jiaming Li for her help in running the samples in mass spectrometry.

\section{Author details}

'Department of Biological Engineering, Massachusetts Institute of Technology, Cambridge, MA 02139, USA. ${ }^{2}$ The Picower Institute for Learning and Memory, Department of Brain and Cognitive Sciences, Massachusetts Institute of Technology, Cambridge, MA 02139, USA. ${ }^{3}$ Department of Chemistry, Massachusetts Institute of Technology, Cambridge, MA 02139, USA

\section{Conflict of interest}

The authors declare that they have no conflict of interest.

\section{Publisher's note}

Springer Nature remains neutral with regard to jurisdictional claims in published maps and institutional affiliations.

Supplementary Information accompanies this paper at (https://doi.org/ 10.1038/s41398-019-0388-7).

Received: 27 September 2018 Revised: 15 December 2018 Accepted: 2 January 2019

Published online: 29 January 2019

\section{References}

1. Lee, V. M., Goedert, M. \& Trojanowski, J. Q. Neurodegenerative tauopathies. Annu. Rev. Neurosci. 24, 1121-1159 (2001).

2. Neve, R. L., Harris, P., Kosik, K. S., Kurnit, D. M. \& Donlon, T. A. Identification of cDNA clones for the human microtubule-associated protein tau and chromosomal localization of the genes for tau and microtubule-associated protein 2. Mol. Brain Res. 1, 271-280 (1986).

3. Cleveland, D. W., Hwo, S.-Y. \& Kirschner, M. W. Physical and chemical properties of purified tau factor and the role of tau in microtubule assembly. J. Mol. Biol. 116, 227-247 (1977).

4. Drubin, D. G. \& Kirschner, M. W. Tau protein function in living cells. J. Cell Biol. 103, 2739-2746 (1986).

5. Billingsley, M. L. \& Kincaid, R. L. Regulated phosphorylation and dephosphorylation of tau protein: effects on microtubule interaction, intracellular trafficking and neurodegeneration. Biochem. J. 323, 577-591 (1997).

6. Iqbal, K. et al. Defective brain microtubule assembly in Alzheimer's disease. Lancet 328, 421-426 (1986).

7. Drechsel, D. N., Hyman, A., Cobb, M. H. \& Kirschner, M. Modulation of the dynamic instability of tubulin assembly by the microtubule-associated protein tau. Mol. Biol. Cell. 3, 1141-1154 (1992).

8. Wolozin, B. L., Pruchnicki, A., Dickson, D. W. \& Davies, P. A neuronal antigen in the brains of Alzheimer patients. Science 232, 648-650 (1986).

9. Morris, M., Maeda, S., Vossel, K. \& Mucke, L. The many faces of tau. Neuron $\mathbf{7 0 ,}$ 410-426 (2011).

10. Dawson, H. N. et al. Inhibition of neuronal maturation in primary hippocampal neurons from t deficient mice. J. Cell Sci. 114, 1179-1187 (2001).

11. Farias, G. A., Muñoz, J. P., Garrido, J. \& Maccioni, R. B. Tubulin, actin, and tau protein interactions and the study of their macromolecular assemblies. J. Cell. Biochem. 85, 315-324 (2002).
12. Guerrero-Muñoz, M. J., Gerson, J. \& Castillo-Carranza, D. L. Tau oligomers: the toxic player at synapses in Alzheimer's disease. Front. Cell. Neurosci. 9, 464 (2015).

13. Nakazawa, T. et al. Characterization of fyn-mediated tyrosine phosphorylation sites on GluRe2 (NR2B) subunit of theN-Methyl-D-aspartate receptor. J. Biol. Chem. 276, 693-699 (2001).

14. Tezuka, T., Umemori, H., Akiyama, T., Nakanishi, S. \& Yamamoto, T. PSD-95 promotes Fyn-mediated tyrosine phosphorylation of the N-methyl-Daspartate receptor subunit NR2A. Proc. Natt. Acad. Sci. 96, 435-440 (1999).

15. Salter, M. W. \& Kalia, L. V. Src kinases: a hub for NMDA receptor regulation. Nat. Rev. Neurosci. 5, 317 (2004)

16. Brenman, J. E. et al. Interaction of nitric oxide synthase with the postsynaptic density protein PSD-95 and a1-syntrophin mediated by PDZ domains. Cell 84, 757-767 (1996).

17. Seneviratne, $U$. et al. S-nitrosation of proteins relevant to Alzheimer's disease during early stages of neurodegeneration. Proc. Natl. Acad. Sci. 113, 4152-4157 (2016).

18. Nakamura, T. et al. Aberrant protein S-nitrosylation contributes to the pathophysiology of neurodegenerative diseases. Neurobiol. Dis. 84, 99-108 (2015).

19. Haun, F. et al. S-nitrosylation of dynamin-related protein 1 mediates mutant huntingtin-induced mitochondrial fragmentation and neuronal injury in Huntington's disease. Antioxid. Redox Signal. 19, 1173-1184 (2013).

20. Chung, K. K., Dawson, V. L. \& Dawson, T. M. S-Nitrosylation in Parkinson's disease and related neurodegenerative disorders. Methods Enzymol. 396, 139-150 (2005).

21. $\mathrm{Qu}$, J. et al. S-Nitrosylation activates $\mathrm{Cdk5}$ and contributes to synaptic spine loss induced by $\beta$-amyloid peptide. Proc. Natl. Acad. Sci. 108, 14330-14335 (2011).

22. Uehara, T. et al. S-nitrosylated protein-disulphide isomerase links protein misfolding to neurodegeneration. Nature 441, 513-517 (2006).

23. Shi, Z-Q. et al. S-nitrosylated SHP-2 contributes to NMDA receptor-mediated excitotoxicity in acute ischemic stroke. Proc. Natl. Acad. Sci. 110, 3137-3142 (2013).

24. Amal, H. et al. Shank3 mutation in a mouse model of autism leads to changes in the S-nitroso-proteome and affects key proteins involved in vesicle release and synaptic function. Mol. Pscychiatry. https://doi.org/10.1038/s41380-0180113-6 (2018).

25. Nakamura, T., Tu, S., Akhtar, M. W. \& Sunico, C. R. Okamoto S-i, Lipton SA. Aberrant protein s-nitrosylation in neurodegenerative diseases. Neuron $\mathbf{7 8}$ 596-614 (2013).

26. Seth, D. et al. A multiplex enzymatic machinery for cellular protein Snitrosylation. Mol. Cell 69, 451-464 (2018).

27. Cruz, J. C. et al. p25/cyclin-dependent kinase 5 induces production and intraneuronal accumulation of amyloid $\beta$ in vivo. J. Neurosci. 26, 10536-10541 (2006).

28. Sperfeld, A. D. et al. FTDP-17: an early-onset phenotype with parkinsonism and epileptic seizures caused by a novel mutation. Ann. Neurol. 46, 708-715 (1999).

29. Yoshiyama, Y. et al. Synapse loss and microglial activation precede tangles in a P301S tauopathy mouse model. Neuron 53, 337-351 (2007).

30. Tseng, H.-C., Zhou, Y., Shen, Y. \& Tsai, L.-H. A survey of Cdk5 activator p35 and p25 levels in Alzheimer's disease brains. FEBS Lett. 523, 58-62 (2002).

31. Seneviratne, U., Godoy, L. C., Wishnok, J. S., Wogan, G. N. \& Tannenbaum, S. R. Mechanism-based triarylphosphine-ester probes for capture of endogenous RSNOs. J. Am. Chem. Soc. 135, 7693-7704 (2013).

32. Rosso, S. B. \& Inestrosa, N. C. WNT signaling in neuronal maturation and synaptogenesis. Front. Cell. Neurosci. 7, 103 (2013).

33. Oliva, C. A., Vargas, J. Y. \& Inestrosa, N. C. Wnts in adult brain: from synaptic plasticity to cognitive deficiencies. Front. Cell. Neurosci. 7, 224 (2013).

34. Komiya, Y. \& Habas, R. Wnt signal transduction pathways. Organogenesis 4 $68-75$ (2008).

35. Chang, J.-Y. et al. CaMKII Autophosphorylation Is Necessary for Optimal Integration of Ca 2+ Signals during LTP Induction, but Not Maintenance. Neuron 94, 800 (2017). 8. e4.

36. Inestrosa, N. C. \& Varela-Nallar, L. Wnt signaling in the nervous system and in Alzheimer's disease. J. Mol. Cell Biol. 6, 64-74 (2014).

37. Wan W., Xia S., Kalionis B., Liu L., Li Y. The role of Wnt signaling in the development of alzheimer's disease: a potential therapeutic target? BioMed research international (2014).

38. Szklarczyk, D. et al. STRINGv10: protein-protein interaction networks, integrated over the tree of life. Nucleic Acids Res. 43, D447-D452 (2015). 
39. Chen, B., Keshive, M. \& Deen, W. M. Diffusion and reaction of nitric oxide in suspension cell cultures. Biophys. J. 75, 745-754 (1998).

40. Allen, B. et al. Abundant tau filaments and nonapoptotic neurodegeneration in transgenic mice expressing human P301S tau protein. J. Neurosci. 22 9340-9351 (2002).

41. Du, A.-T. et al. Different regional patterns of cortical thinning in Alzheimer's disease and frontotemporal dementia. Brain 130, 1159-1166 (2007).

42. Fjell, A. M. et al. The roots of Alzheimer's disease: are high-expanding cortical areas preferentially targeted? Cereb. Cortex. 25, 2556-2565 (2014).

43. Agster, K. L. \& Burwell, R. D. Hippocampal and subicular efferents and afferents of the perirhinal, postrhinal, and entorhinal cortices of the rat. Behav. brain Res. 254, 50-64 (2013)

44. Scholz, B. et al. Endothelial RSPO3 controls vascular stability and pruning through non-canonical WNT/Ca 2+/NFAT signaling. Dev. Cell. 36, 79-93 (2016).

45. Wille, P. C. \& Gross, S. S. Regulation of the E3 ubiquitin ligase RNF25 by nitric oxide. Nitric Oxide. 27, S38 (2012).

46. Yao, D. et al. Nitrosative stress linked to sporadic Parkinson's disease: Snitrosylation of parkin regulates its E3 ubiquitin ligase activity. Proc. Natl. Acad. Sci. USA. 101, 10810-10814 (2004).

47. Small, D. H. Dysregulation of calcium homeostasis in Alzheimer's disease. Neurochem. Res. 34, 1824-1829 (2009).

48. Popugaeva, E., Pchitskaya, E. \& Bezprozvanny, I. Dysregulation of neuronal calcium homeostasis in Alzheimer's disease-A therapeutic opportunity? Biochem. Biophys. Res. Commun. 483, 998-1004 (2017).

49. Hollands, C., Bartolotti, N. \& Lazarov, O. Alzheimer's disease and hippocampal adult Neurogenesis; exploring shared mechanisms. Front. Neurosci. 10, 178 (2016).

50. Tampellini, D. Synaptic activity and Alzheimer's disease: a critical update. Front. Neurosci. 9, 423 (2015)

51. Cataldo, A. M. et al. Endocytic pathway abnormalities precede amyloid $\beta$ deposition in sporadic Alzheimer's disease and Down syndrome: differential effects of APOE genotype and presenilin mutations. Am. J. Pathol. 157, 277-286 (2000).

52. Nixon, R. A. Endosome function and dysfunction in Alzheimer's disease and other neurodegenerative diseases. Neurobiol. Aging 26, 373-382 (2005).

53. Fischer, A., Sananbenesi, F., Pang, P. T., Lu, B. \& Tsai, L.-H. Opposing roles of transient and prolonged expression of p25 in synaptic plasticity and hippocampus-dependent memory. Neuron 48, 825-838 (2005).
54. Takeuchi, H. et al. P301S mutant human tau transgenic mice manifest early symptoms of human tauopathies with dementia and altered sensorimotor gating. Plos One 6, e21050 (2011).

55. McQuate, A., Latorre-Esteves, E. \& Barria, A. A Wnt/calcium signaling cascade regulates neuronal excitability and trafficking of NMDARs. Cell Rep. 21, 60-69 (2017).

56. Hess, D. T. \& Stamler, J. S. Regulation by S-nitrosylation of protein posttranslational modification. J. Biol. Chem. 287, 4411-4418 (2012).

57. Andersson, D. C. et al. Ryanodine receptor oxidation causes intracellular calcium leak and muscle weakness in aging. Cell Metab. 14, 196-207 (2011).

58. Nguyen, T. \& Di Giovanni, S. NFAT signaling in neural development and axon growth. Int. J. Dev. Neurosci. 26, 141-145 (2008).

59. Yoshimura, Y., Ichinose, T. \& Yamauchi, T. Phosphorylation of tau protein to sites found in Alzheimer's disease brain is catalyzed by Ca2+/calmodulindependent protein kinase $\|$ as demonstrated tandem mass spectrometry. Neurosci. Lett. 353, 185-188 (2003).

60. Ghosh, A. \& Giese, K. P. Calcium/calmodulin-dependent kinase II and Alzheimer's disease. Mol. brain. 8, 78 (2015).

61. Oka, M. et al. Ca2+/calmodulin-dependent protein kinase II promotes neurodegeneration caused by tau phosphorylated at Ser262/356 in a transgenic Drosophila model of tauopathy. J. Biochem. 162, 335-342 (2017).

62. Abdul, H. M., Furman, J. L., Sama, M. A., Mathis, D. M. \& Norris, C. M. NFATs and Alzheimer's disease. Mol. Cell. Pharmacol. 2, 7 (2010).

63. Cho, H. J., Jin, S. M., Youn, H. D., Huh, K. \& Mook-Jung, I. Disrupted intracellular calcium regulates BACE1 gene expression via nuclear factor of activated $T$ cells 1 (NFAT 1) signaling. Aging Cell. 7, 137-147 (2008).

64. Burns, L. H. \& Wang, H.-Y. Altered filamin A enables amyloid beta-induced tau hyperphosphorylation and neuroinflammation in Alzheimer's disease. Neuroimmunol. Neuroinflamm. 4, 263-271 (2017).

65. Wang, H.Y. et al. Reducing amyloid-related Alzheimer's disease pathogenesis by a small molecule targeting filamin A. J. Neurosci. 32, 9773-9784 (2012)

66. Pohanka, M. Alzheimer s disease and oxidative stress: a review. Curr. Med. Chem. 21, 356-364 (2014).

67. Tönnies, E. \& Trushina, E. Oxidative stress, synaptic dysfunction, and Alzheimer's disease. J. Alzheimer's Dis. 57, 1105-1121 (2017).

68. Bredt, D. S., Hwang, P. M. \& Snyder, S. H. Localization of nitric oxide synthase indicating a neural role for nitric oxide. Nature 347, 768 (1990). 